\title{
Simultaneous Nodal Superconductivity and Time-Reversal Symmetry Breaking in the Noncentrosymmetric Superconductor CaPtAs
}

\author{
T. Shang® ${ }^{1,2, *}$ M. Smidman, ${ }^{3}$ A. Wang, ${ }^{3}$ L.-J. Chang $\odot,{ }^{4}$ C. Baines, ${ }^{5}$ M. K. Lee, ${ }^{4}$ Z. Y. Nie, ${ }^{3}$ G. M. Pang, ${ }^{3}$ W. Xie, ${ }^{3}$ \\ W. B. Jiang, ${ }^{3}$ M. Shi, ${ }^{6}$ M. Medarde, ${ }^{1}$ T. Shiroka ${ }^{5,7}$ and H. Q. Yuan ${ }^{3,8, \dagger}$ \\ ${ }^{1}$ Laboratory for Multiscale Materials Experiments, Paul Scherrer Institut, Villigen CH-5232, Switzerland \\ ${ }^{2}$ Physik-Institut, Universität Zürich, Winterthurerstrasse 190, Zürich CH-8057, Switzerland \\ ${ }^{3}$ Center for Correlated Matter and Department of Physics, Zhejiang University, Hangzhou 310058, China \\ ${ }^{4}$ Department of Physics, National Cheng Kung University, Tainan 70101, Taiwan \\ ${ }^{5}$ Laboratory for Muon-Spin Spectroscopy, Paul Scherrer Institut, Villigen PSI CH-5232, Switzerland \\ ${ }^{6}$ Swiss Light Source, Paul Scherrer Institut, Villigen CH-5232, Switzerland \\ ${ }^{7}$ Laboratorium für Festkörperphysik, ETH Zürich, Zürich CH-8093, Switzerland \\ ${ }^{8}$ Collaborative Innovation Center of Advanced Microstructures, Nanjing Univeristy, Nanjing 210093, China
}

(Received 16 December 2019; revised manuscript received 12 March 2020; accepted 21 April 2020; published 18 May 2020)

\begin{abstract}
By employing a series of experimental techniques, we provide clear evidence that CaPtAs represents a rare example of a noncentrosymmetric superconductor which simultaneously exhibits nodes in the superconducting gap and broken time-reversal symmetry (TRS) in its superconducting state (below $\left.T_{c} \approx 1.5 \mathrm{~K}\right)$. Unlike in fully gapped superconductors, the magnetic penetration depth $\lambda(T)$ does not saturate at low temperatures, but instead it shows a $T^{2}$ dependence, characteristic of gap nodes. Both the superfluid density and the electronic specific heat are best described by a two-gap model comprising of a nodeless gap and a gap with nodes, rather than by single-band models. At the same time, zero-field muonspin relaxation spectra exhibit increased relaxation rates below the onset of superconductivity, implying that TRS is broken in the superconducting state of CaPtAs, hence indicating its unconventional nature. Our observations suggest $\mathrm{CaPtAs}$ to be a new remarkable material that links two apparently disparate classes, that of TRS-breaking correlated magnetic superconductors with nodal gaps and the weakly correlated noncentrosymmetric superconductors with broken TRS, normally exhibiting only a fully gapped behavior.
\end{abstract}

DOI: 10.1103/PhysRevLett.124.207001

When entering the superconducting state, the breaking of extra symmetries in addition to $\mathrm{U}(1)$ gauge symmetry is normally an indication of unconventional superconductivity (SC) $[1,2]$. In a growing number of superconductors, time-reversal symmetry (TRS) breaking has been proved via the detection of spontaneous magnetic fields below the onset of superconductivity by means of zero-field muon-spin relaxation measurements. Notable examples include $\mathrm{Sr}_{2} \mathrm{RuO}_{4}$ [3], $\mathrm{UPt}_{3}$ [4], $\mathrm{PrOs}_{4} \mathrm{Sb}_{4}$ [5], $\mathrm{LaNiGa}_{2}$ [6], $\mathrm{LaNiC}_{2}, \mathrm{La}_{7} T_{3}$, and $\operatorname{Re} T(T=$ transition metal $)$ superconductors [7-13]. The first two are well-known examples of strongly correlated superconductors with unconventional pairing mechanisms [14,15], while the latter three are examples of noncentrosymmetric superconductors (NCSCs), where the lack of inversion symmetry gives rise to an antisymmetric spin-orbit coupling (ASOC) leading to spin-split Fermi surfaces. Consequently, their pairing states are not constrained to be purely singlet or triplet, and mixed-parity pairing may occur [16-18]. Owing to such mixed pairing and/or the influence of ASOC, NCSCs may exhibit significantly different properties from their conventional counterparts, e.g., superconducting gaps with nodes [19-23], upper critical fields exceeding the
Pauli limit [11,24-27] or, as recently proposed, even topological superconductivity [28-32].

In general, the relationship between the breaking of TRS and a lack of inversion symmetry in the crystal structure is unclear. In many NCSCs such as $\mathrm{Mo}_{3} \mathrm{Al}_{2} \mathrm{C}, \mathrm{LaTSi}_{3}$, $\mathrm{Mg}_{10} \mathrm{Ir}_{19} \mathrm{~B}_{16}$, or $\mathrm{Mo}_{3} \mathrm{P}$ [33-38], no spontaneous magnetic fields have been observed and thus TRS is preserved in the superconducting state. A notable feature of most of the weakly correlated NCSCs with broken TRS is the presence of fully opened superconducting gaps. In the case of $\mathrm{LaNiC}_{2}$, inconsistent results, including both fully opened and nodal gap structures, have been found from measurements of the order parameter [39-44]. The nodeless superconductivity of weakly correlated NCSCs is not only in contrast to the general expectations for strong singlettriplet mixing, but also sets these systems apart from the strongly correlated superconductors $\mathrm{Sr}_{2} \mathrm{RuO}_{4}$ and $\mathrm{UPt}_{3}$ $[3,4]$, where the presence of unconventional pairing mechanisms is more unambiguously determined.

In $\mathrm{LaNiC}_{2}$, as well as in centrosymmetric $\mathrm{LaNiGa}_{2}$, the observed TRS breaking has been accounted for by nonunitary triplet pairing $[6,7,45]$. This was reconciled with nodeless multigap SC by the proposal of even-parity triplet 
pairing, between electrons on different orbitals [46]. On the other hand, the $\operatorname{Re} T$ superconductors, which have a relatively large ASOC compared to $\mathrm{LaNiC}_{2}$, appear to exhibit single fully opened gaps, more consistent with a predominantly singlet pairing. The recent observation of TRS breaking in centrosymmetric elemental Re strongly suggests that the local electronic structure of Re is crucial for understanding the TRS breaking in the Re $T$ family [12]. The broken TRS in weakly correlated systems, which otherwise appear to behave as conventional superconductors, has led to proposals to account for this behavior with a conventional pairing mechanism [47], such as the loop-Josephson-current state, based on a model with onsite singlet pairing [48].

To date, there are scarcely any examples of NCSC which clearly exhibit broken TRS and nodal-gap SC. In this Letter, we show that CaPtAs, a newly discovered NCSC [49], is a rare candidate to display both such unconventional features. Our key observations of a nodal-gap and of spontaneous magnetic fields (concomitant with the onset of $\mathrm{SC}$ ) indicate that CaPtAs represents a new remarkable example of weakly correlated NCSC encompassing both broken TRS and nodal SC.

Polycrystalline CaPtAs was synthesized via a solid-state reaction method [49]. Magnetic susceptibility, electrical resistivity, and specific-heat measurements were performed on a Quantum Design MPMS and PPMS, respectively. The muon-spin relaxation and rotation $(\mu \mathrm{SR})$ measurements were carried out on the low-temperature facility (LTF) spectrometer of the $\pi \mathrm{M} 3$ beam line at the Paul Scherrer Institut, Villigen, Switzerland. The temperature-dependent shift of the magnetic penetration depth, which is proportional to the frequency shift, i.e., $\Delta \lambda=G \Delta f$ (with $G$ a geometry related constant), was measured by using a tunnel-diode oscillator (TDO)-based technique at an operating frequency of $7 \mathrm{MHz}[22,27,40]$.

CaPtAs crystallizes in a tetragonal noncentrosymmetric structure with space group $I 4_{1} m d$ (No. 109) [49]. The SC of CaPtAs was characterized by magnetic susceptibility, measured using both field-cooling (FC) and zero-fieldcooling (ZFC) protocols. As shown in Fig. 1(a), the ZFC susceptibility (after accounting for the demagnetization factor) indicates $\mathrm{SC}$ below $T_{c}=1.5 \mathrm{~K}$, where the electrical resistivity (right axis) drops to zero, both being consistent with the specific-heat data [50]. The lower critical field, estimated from the field-dependent magnetization, is $\mu_{0} H_{c 1}=4.8(1) \mathrm{mT}$ [see Fig. 1(b)].

Figure 2 shows the temperature dependent magnetic penetration depth $\lambda(T)$ measured by the TDO method and the corresponding exponential- and power-law fits. The TDO data over the full temperature range (see inset) illustrate the superconducting transition near $T_{c}=1.5 \mathrm{~K}$. Clearly, $\lambda(T)$ follows a quadratic temperature dependence $\left[\lambda(T) \sim T^{2}\right]$, as expected for superconductors with point nodes. In contrast, a power-law with a larger exponent $\left[\lambda(T) \sim T^{3}\right]$ or an exponential temperature dependence
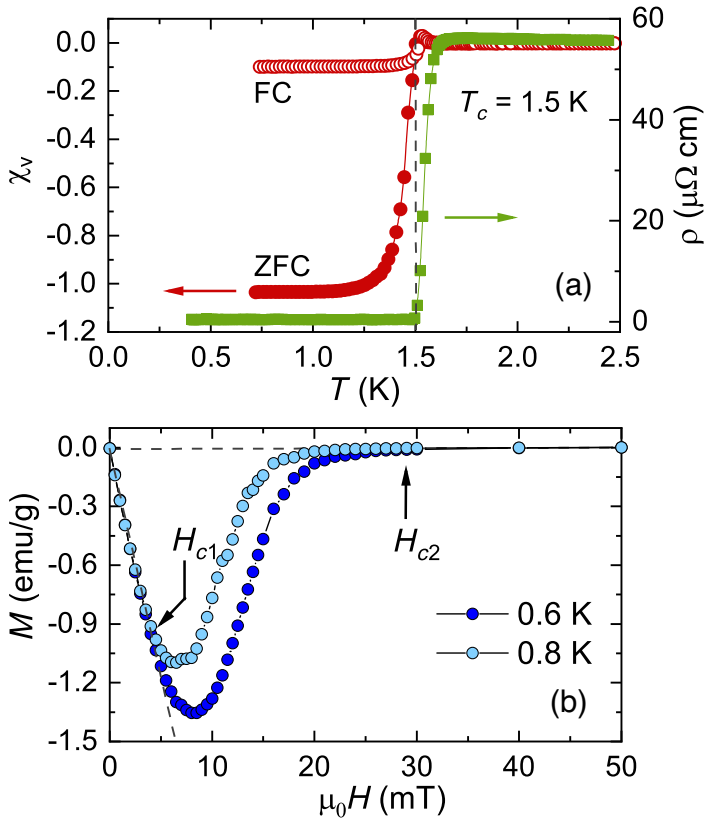

FIG. 1. (a) Temperature dependent ZFC- and FC-magnetic susceptibilities of CaPtAs (left axis), measured in an applied field of $1 \mathrm{mT}$, and zero-field electrical resistivity (right axis). The magnetic susceptibility data were corrected after considering the demagnetization factor. (b) Magnetization vs applied magnetic field in the superconducting state. The lower critical field $H_{c 1}$ was determined as the value where $M(H)$ starts deviating from linearity (see dashed line), while the upper critical field $H_{c 2}$ was identified as the field where the diamagnetic signal disappears.

$\left[\lambda(T) \sim e^{-\Delta_{0}(0) / T}\right]$, the latter indicating fully gapped behavior, both deviate significantly from the experimental data.

Figure 3(a) shows two typical transverse-field (TF) $\mu$ SR spectra collected at temperatures above and below $T_{c}$ at

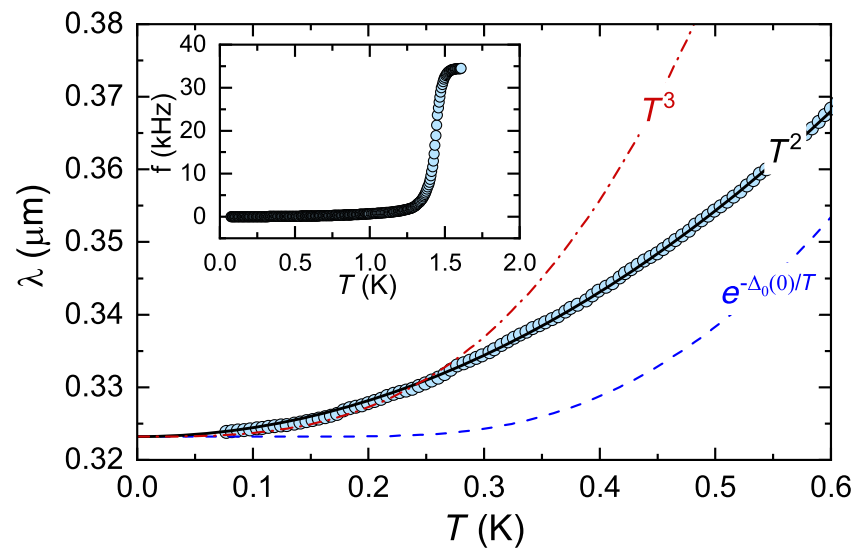

FIG. 2. The magnetic penetration depth $\lambda(T)$ of CaPtAs, measured using the TDO-based method in zero field at $T \lesssim 1 / 3 T_{c}$. The inset shows the TDO frequency up to temperatures above $T_{c}$. The black and red lines represent fits to $\lambda(T) \sim T^{2}$ and $T^{3}$, respectively, while the blue line indicates an exponential temperature dependence. $\lambda$ was calculated as $\lambda_{0}+\Delta \lambda$, where $\lambda_{0}$ was derived from $\mathrm{TF}-\mu \mathrm{SR}$ measurements. 

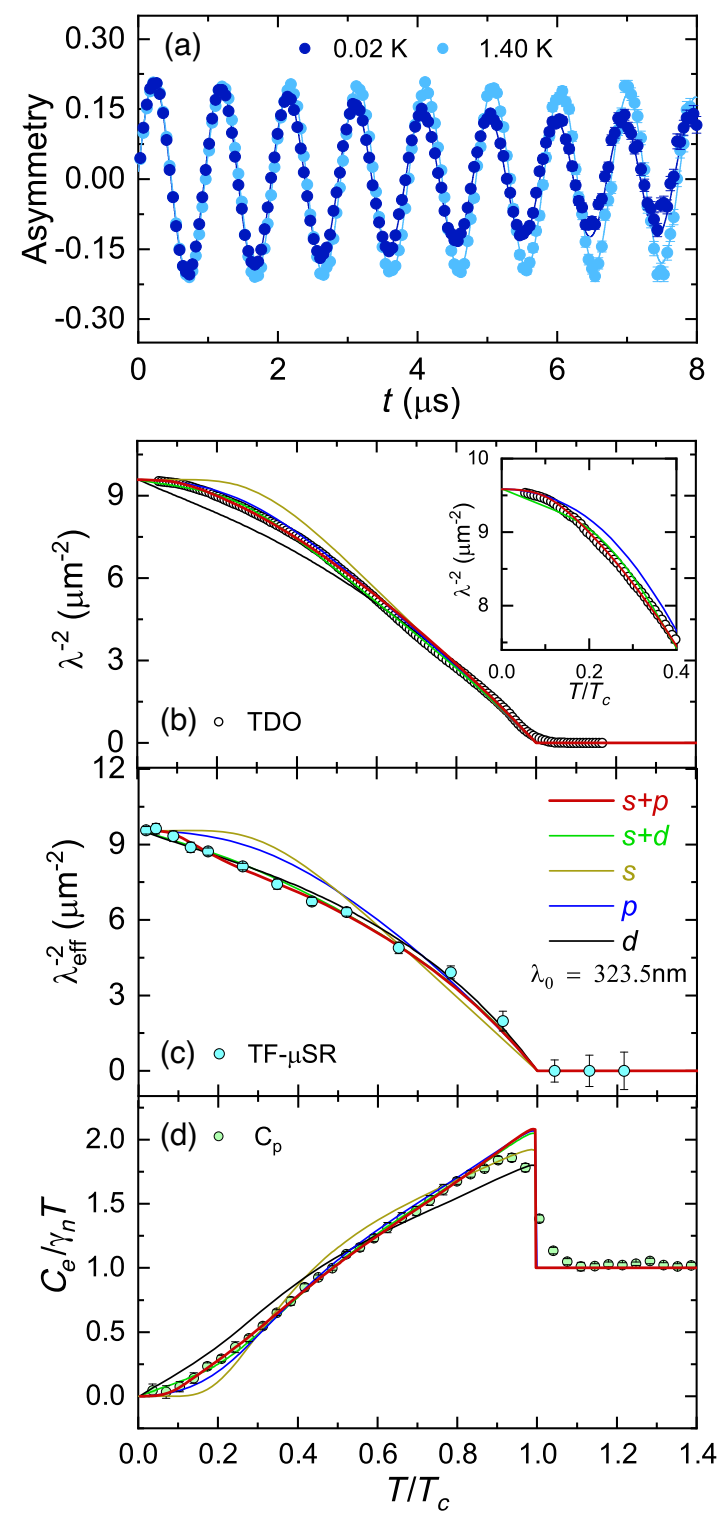

FIG. 3. (a) Time-domain TF- $\mu \mathrm{SR}$ spectra in the superconducting $(0.02 \mathrm{~K})$ and normal $(1.4 \mathrm{~K})$ states of CaPtAs. Superfluid density as estimated from (b) The TDO-based method and (c) $\mathrm{TF}-\mu \mathrm{SR}$ vs the reduced temperature $T / T_{c}$. The inset shows the enlarged plot of the TDO low- $T$ region. (d) Zero-field electronic specific heat vs $T / T_{c}$. The different lines represent fits to the various models (see text for details). The fit parameters are listed in Table SI [50].

$8 \mathrm{mT}\left(T_{c}^{8 \mathrm{mT}}=1.15 \mathrm{~K}\right)$ [50], here corresponding to nearly twice $\mu_{0} H_{c 1}(0)$. The TF- $\mu$ SR asymmetry data were analyzed using:

$A_{\mathrm{TF}}=A_{s} e^{-\sigma^{2} t^{2} / 2} \cos \left(\gamma_{\mu} B_{s} t+\phi\right)+A_{\mathrm{bg}} \cos \left(\gamma_{\mu} B_{\mathrm{bg}} t+\phi\right)$.

Here $A_{s}(50 \%)$ and $A_{\mathrm{bg}}(50 \%)$ represent the asymmetry of the sample and background (e.g., sample holder), respectively. $\gamma_{\mu} / 2 \pi=135.53 \mathrm{MHz} / \mathrm{T}$ is the muon gyromagnetic ratio, $B_{s}$ and $B_{\mathrm{bg}}$ are the local fields sensed by implanted muons in the sample and sample holder, $\phi$ is the shared initial phase, and $\sigma$ is the Gaussian relaxation rate. $\sigma$ includes contributions from both the flux-line lattice $\left(\sigma_{\mathrm{sc}}\right)$ and a temperature-invariant relaxation due to nuclear moments $\left(\sigma_{n}\right)$. By subtracting the nuclear contribution in quadrature, one can extract $\sigma_{\mathrm{sc}}$, i.e., $\sigma_{\mathrm{sc}}=\sqrt{\sigma^{2}-\sigma_{n}^{2}}$. The upper critical field of CaPtAs is relatively small compared to the field applied during the TF- $\mu \mathrm{SR}$ measurements $\left(H_{c 2} / H_{\mathrm{appl}} \sim\right.$ 4.3) [50-52]. Hence, the effective penetration depth $\lambda_{\text {eff }}$ had to be calculated from $\sigma_{\mathrm{sc}}$ by considering the overlap of the vortex cores [53]:

$\sigma_{\mathrm{sc}}(h)=0.172 \frac{\gamma_{\mu} \Phi_{0}}{2 \pi}(1-h)\left[1+1.21(1-\sqrt{h})^{3}\right] \lambda_{\text {eff }}^{-2}$.

Here, $h=H_{\text {appl }} / H_{c 2}$, is the reduced magnetic field.

Figures 3(b)-3(c) show the superfluid density $\left(\rho_{\mathrm{sc}} \propto \lambda^{-2}\right)$ measured by TDO and $\mu \mathrm{SR}$ vs the reduced temperature $T / T_{c}$, respectively. The superfluid density clearly varies with temperature down to the lowest $T$, i.e., well below $T / T_{c}=0.3$. This nonconstant behavior again indicates the presence of low energy excitations and, hence, of nodes in the superconducting gap. To get further insight into the pairing symmetry, the temperature-dependent superfluid density was analyzed using different models. Considering a superconducting gap $\Delta_{k}$, the superfluid density $\rho_{\mathrm{sc}}(T)$ can be calculated as:

$$
\rho_{\mathrm{sc}}=1+2\left\langle\int_{\Delta_{\mathrm{k}}}^{\infty} \frac{E}{\sqrt{E^{2}-\Delta_{\mathrm{k}}^{2}}} \frac{\partial f}{\partial E} d E\right\rangle_{\mathrm{FS}},
$$

where $f=\left(1+e^{E / k_{B} T}\right)^{-1}$ is the Fermi function and \langle\rangle$_{\mathrm{FS}}$ represents an average over the Fermi surface. The gap function can be written as $\Delta_{k}(T)=\Delta_{0}(T) g_{k}$, where $\Delta_{0}$ is the maximum gap value and $g_{k}$ is the angular dependence of the gap (see details in Table SI) [50]. The temperature dependence of the gap was assumed to follow $\Delta_{0}(T)=$ $\Delta_{0}(0) \tanh \left\{1.82\left[1.018\left(T_{c} / T-1\right)\right]^{0.51}\right\}$, where $\Delta_{0}(0)$ is the gap value in the zero-temperature limit.

Five different models, single-gap $s, p, d$, and two-gap $s+p$ and $s+d$ wave, were used to analyze the superfluid density. The marked temperature dependence of the superfluid density at low- $T$ clearly rules out a fully gapped $s$-wave model [see yellow lines in Figs. 3(b)-3(c)]. Also in the case of a pure $p$ wave, we find a poor agreement with the low- $T$ data (blue lines). A $d$-wave model with line nodes, can reproduce reasonably well the TF- $\mu$ SR data, but it fails to follow the low- $T \lambda^{-2}(T)$ data obtained via TDO [see black lines in Figs. 3(b)-3(c)]. The slight difference between the TDO and TF- $\mu$ SR data below $T / T_{c} \sim 0.5$ is most likely due to the applied external field $(8 \mathrm{mT})$ during the TF- $\mu$ SR measurements, which is not negligible compared to the small $H_{c 2}$ value of CaPtAs.

Conversely, the superfluid density is best fitted by a twocomponent $(s+p)$ - or $(s+d)$-wave model [red and green 
lines in Figs. 3(b)-3(c)]. The good agreement with data of these models indicates the presence of multiple gaps, of which at least one has nodes on the Fermi surface. Although both models fit the superfluid density satisfactorily well across the full temperature range $\left(T<T_{c}\right)$, the $(s+p)$-wave model agrees better with the $\lambda^{-2}(T)$ data measured using the TDO method [see inset of Fig. 3(b)]. This is also strongly evidenced by both its smaller deviation from the data (see Table SI) and the quadratic low- $T$ dependence of $\lambda(T)$ [50].

To further validate the above conclusions, the zero-field electronic specific heat $C_{e} / T$ was also analyzed using the above models $[51,54,55]$. $C_{e} / T$ was obtained by subtracting the phonon- and nuclear contributions from the measured data (see Fig. S2) [50] and it is shown in Fig. 3(d) as $C_{e} / \gamma_{n} T$, with $\gamma_{n}$ the normal-state electronic specific-heat coefficient. Again, the single-gap $s$-, $p$-, and $d$-wave models deviate significantly from the data. Conversely, both multigap models exhibit a good agreement with the experimental data across the full temperature range, with the $(s+p)$-wave model showing the smallest deviation [50], hence providing further evidence for nodal-gap SC in CaPtAs. The fit of the $(s+p)$-wave model to the superfluid density and the electronic specific heat [including the $s$ $(\sim 15 \%)$ and $p$-wave $(\sim 85 \%)$ components] are shown in Fig. S3 [50].

To search for spontaneous fields below $T_{c}$, signaling possible TRS breaking in CaPtAs, we performed zero field (ZF) $\mu \mathrm{SR}$ measurements. The clear increase in relaxation rate in the superconducting state [see Fig. 4(a)] hints at the breaking of TRS. For nonmagnetic materials, the depolarization is generally described by a Gaussian KuboToyabe relaxation function [56,57]. For CaPtAs, the $\mathrm{ZF}-\mu \mathrm{SR}$ spectra were fitted by considering an additional Lorentzian relaxation component, with $A_{s}$ and $A_{\mathrm{bg}}$ being the same as in the TF- $\mu$ SR case:

$A_{\mathrm{ZF}}=A_{s}\left\{\frac{1}{3}+\frac{2}{3}\left(1-\sigma_{\mathrm{ZF}}^{2} t^{2}\right) e^{\left.\left[-\left(\sigma_{\mathrm{ZF}}^{2} t^{2}\right) / 2\right)\right]}\right\} e^{-\Lambda_{\mathrm{ZF}} t}+A_{\mathrm{bg}}$.

Fits using the above model yield an almost temperatureindependent Gaussian relaxation rate $\left(\sigma_{\mathrm{ZF}}\right)$ across the measured temperature range [see Fig. S4(b)] [50]. Hence, the Lorentzian relaxation rate $\left(\Lambda_{\mathrm{ZF}}\right)$ was estimated by fixing $\sigma_{\mathrm{ZF}}$ to its average value $\left(\sigma_{\mathrm{ZF}}^{\text {avg }}=0.13 \mu \mathrm{s}^{-1}\right)$. As shown in Fig. 4(b), a small yet measurable increase of $\Lambda_{\mathrm{ZF}}$ below $T_{c}$ and a temperature-independent relaxation above $T_{c}$ reflect the onset of spontaneous magnetic fields. This can be considered as the signature of TRS breaking in the superconducting state of CaPtAs, with similarly enhanced $\Lambda_{\mathrm{ZF}}$ having also been found in other TRS breaking NCSCs $[7,8]$. Both free- and fixed- $\sigma_{\mathrm{ZF}}$ analyses show a robust increase in $\Lambda_{\mathrm{ZF}}(T)$ below $T_{c}$, demonstrating that the signal of spontaneous magnetic fields is an intrinsic effect, rather
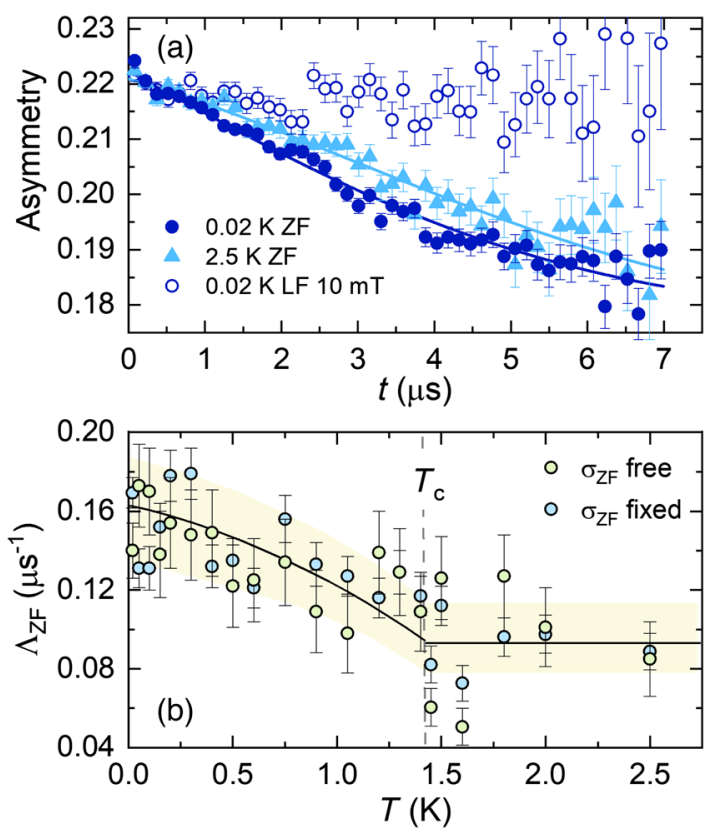

FIG. 4. (a) Representative zero-field $\mu$ SR time spectra collected in the superconducting $(0.02 \mathrm{~K})$ and normal $(2.5 \mathrm{~K})$ state of CaPtAs. Additional data were collected at $0.02 \mathrm{~K}$ in a $10-\mathrm{mT}$ longitudinal field. The solid lines are fits to Eq. (4). (b) Derived Lorentzian relaxation rate $\Lambda_{\mathrm{ZF}}$ (using either a free- or a fixed- $\sigma_{\mathrm{ZF}}$ analysis) versus temperature. The solid line through the data is a guide to the eyes.

than an artifact of correlated fit parameters. This is further confirmed in Fig. S5, where we show the cross correlations between the different fit parameters [50]. Finally, longitudinal-field (LF) $\mu \mathrm{SR}$ measurements were performed at base temperature $(0.02 \mathrm{~K})$ to rule out additional extrinsic effects such as defect or impurity induced relaxation. As shown in Fig. 4(a), a small field of $10 \mathrm{mT}$ is sufficient to fully decouple the muon spins from the weak spontaneous magnetic fields, indicating that the fields are static on the timescale of the muon lifetime.

To date, most NCSCs with broken TRS exhibit nodeless superconductivity, indicating that the spin-singlet channel dominates the pairing. These include the $\alpha$-Mn-type $\operatorname{Re} T$ [10-13] and $\mathrm{Th}_{7} \mathrm{Fe}_{3}$-type $\mathrm{La}_{7} T_{3}[8,9]$. As for $\mathrm{CeNiC}_{2}$-type NCSCs, the recently discovered $\mathrm{ThCoC}_{2}$ exhibits nodal SC, but no evidence of broken TRS has been found [58]. In $\mathrm{LaNiC}_{2}$, the low symmetry of its orthorhombic crystal structure means that the breaking of TRS at $T_{c}$ necessarily implies nonunitary triplet pairing, and rules out the mixed singlet-triplet state described below [7,45]. However, measurements of the gap symmetry have yielded inconsistent results, where both fully-opened and nodal gap structures have been reported $[39,40,40-43]$. Compared to the above cases, CaPtAs represents a new remarkable NCSC, which accommodates both broken TRS and nodal SC.

One possibility is that the observed multigap superconductivity corresponds to different gaps on distinct 
electronic bands, which would be consistent with band structure calculations showing multiple bands crossing the Fermi level [49]. An alternative scenario is that in NCSCs, the admixture of singlet $\psi(\boldsymbol{k})$ and triplet $\boldsymbol{d}(\boldsymbol{k})$ order parameters leads to gap structures with $\Delta(\boldsymbol{k})_{ \pm}=$ $\psi(\boldsymbol{k}) \pm|\boldsymbol{d}(\boldsymbol{k})|$ [16-18]. Clearly, if the triplet component is small, the gaps on the spin-split Fermi surfaces will both be nodeless and of nearly equal magnitude, making this case hardly distinguishable from a single-gap $s$-wave superconductor. On the other hand, if the triplet component dominates, there can be one nodeless gap, and another with nodes. Such a scenario can explain well, for instance, the multigap nodeless $\mathrm{SC}$ in $\mathrm{Li}_{2} \mathrm{Pd}_{3} \mathrm{~B}$ and the nodal $\mathrm{SC}$ in $\mathrm{Li}_{2} \mathrm{Pt}_{3} \mathrm{~B}$, since the weight of the triplet-component increases with the enhanced ASOC upon the substitution of Pt for Pd [19]. We find that the superfluid density of CaPtAs is best described by models with one nodeless and one nodal gap, which also corresponds to that expected for significant singlet-triplet mixing.

According to band structure calculations, the estimated band splitting due to ASOC is about $50-100 \mathrm{meV}$, which gives $E_{\mathrm{soc}} / k_{B} T_{c} \sim 400-800$ [49]. Though much smaller than the band splitting of $\mathrm{CePt}_{3} \mathrm{Si}\left(E_{\mathrm{soc}} / k_{B} T_{c} \sim 3095\right)$ [59], it is comparable to that of $\mathrm{Li}_{2} \mathrm{Pt}_{3} \mathrm{~B}(\sim 831)$ [60], and much larger than that of most other NCSCs. Since the above two Pt compounds are believed to exhibit mixed pairing $[19,61]$, the presence of large band splitting due to ASOC, in addition to nodal multigap SC, suggests that $\mathrm{CaPtAs}$ is a good candidate for large singlet-triplet mixing. However, whether in this crystal structure there is a TRS-breaking mixed singlet-triplet state compatible with the ASOC, requires further theoretical analysis. We note that, if one considers TRS breaking states corresponding to the twodimensional irreducible representations of the point group $C_{4 v}$, the simplest one is a chiral $p$-wave state [18], originally applied to $\mathrm{Sr}_{2} \mathrm{RuO}_{4}$ [14]. The $p$-wave model and the $p$-component of $(s+p)$-wave model we use in Fig. 3 correspond to this chiral $p$-wave state. A specific attribution of the pairing symmetry requires further measurements on single crystals, together with microscopic calculations based on the band structure. Different from the proposed topological NCSCs [28,29], CaPtAs exhibits simultaneously nodal SC and broken TRS. This suggests that it could possibly be an exotic type of topological superconductor, a suitable candidate material in which to search for Majorana zero modes [62,63]. Due to its high stability and the availability of single crystals [49], CaPtAs is very promising for future investigations using other techniques, such as scanning tunneling microscopy (STM), or angle-resolved photoemission spectroscopy (ARPES).

In summary, we find that CaPtAs is an example of an NCSC exhibiting both TRS breaking and nodal superconductivity. Its superfluid density and specific heat are best described by a two-gap model, with one gap being fully open and the other being nodal. The presence of multigap nodal superconductivity and sizeable band splitting due to ASOC makes CaPtAs a good candidate for hosting mixed singlet and triplet pairing. While further theoretical calculations and measurements are necessary to determine the nature of the order parameter and pairing mechanism, this system may offer new insights for bridging the gap between different classes of TRS-breaking superconductors, namely strongly correlated superconductors with magnetically mediated pairing and nodal gaps (such as $\mathrm{Sr}_{2} \mathrm{RuO}_{4}$ and $\mathrm{UPt}_{3}$ ) and the more recently discovered weakly correlated NCSCs.

This work was supported by the National Key R\&D Program of China (Grants No. 2016YFA0300202 and No. 2017YFA0303100), the National Natural Science Foundation of China (Grants No. U1632275, No. 11874320, and No. 11974306), the Sino-Swiss Science and Technology Cooperation (Grant No. IZLCZ2-170075), the Schweizerische Nationalfonds zur Förderung der Wissenschaftlichen Forschung, SNF (Grants No. 200021-169455 and No. 206021-139082). L. J.C. thanks the MOST Funding of Taiwan for the support under the Projects No. 104-2112-M-006-010MY3 and No. 107-2112-M-006-020. We also acknowledge the assistance from other beam line scientists on LTF $\mu \mathrm{SR}$ spectrometer at PSI.

*Corresponding authors.

tian.shang@psi.ch

hqyuan@zju.edu.cn

[1] M. Sigrist and K. Ueda, Phenomenological theory of unconventional superconductivity, Rev. Mod. Phys. 63, 239 (1991).

[2] C. C. Tsuei and J. R. Kirtley, Pairing symmetry in cuprate superconductors, Rev. Mod. Phys. 72, 969 (2000).

[3] G. M. Luke, Y. Fudamoto, K. M. Kojima, M. I. Larkin, J. Merrin, B. Nachumi, Y. J. Uemura, Y. Maeno, Z. Q. Mao, Y. Mori, H. Nakamura, and M. Sigrist, Time-reversal symmetry-breaking superconductivity in $\mathrm{Sr}_{2} \mathrm{RuO}_{4}$, Nature (London) 394, 558 (1998).

[4] G. M. Luke, A. Keren, L. P. Le, W. D. Wu, Y. J. Uemura, D. A. Bonn, L. Taillefer, and J. D. Garrett, Muon Spin Relaxation in $\mathrm{UPt}_{3}$, Phys. Rev. Lett. 71, 1466 (1993).

[5] Y. Aoki, A. Tsuchiya, T. Kanayama, S. R. Saha, H. Sugawara, H. Sato, W. Higemoto, A. Koda, K. Ohishi, K. Nishiyama, and R. Kadono, Time-Reversal SymmetryBreaking Superconductivity in Heavy-Fermion $\mathrm{PrOs}_{4} \mathrm{Sb}_{12}$ Detected by Muon-Spin Relaxation, Phys. Rev. Lett. 91, 067003 (2003).

[6] A. D. Hillier, J. Quintanilla, B. Mazidian, J. F. Annett, and R. Cywinski, Nonunitary Triplet Pairing in the Centrosymmetric Superconductor $\mathrm{LaNiGa}_{2}$, Phys. Rev. Lett. 109, 097001 (2012).

[7] A. D. Hillier, J. Quintanilla, and R. Cywinski, Evidence for Time-Reversal Symmetry Breaking in the Noncentrosymmetric Superconductor $\mathrm{LaNiC}_{2}$, Phys. Rev. Lett. 102, 117007 (2009). 
[8] J. A. T. Barker, D. Singh, A. Thamizhavel, A. D. Hillier, M. R. Lees, G. Balakrishnan, D. M. Paul, and R. P. Singh, Unconventional Superconductivity in $\mathrm{La}_{7} \mathrm{Ir}_{3}$ Revealed by Muon Spin Relaxation: Introducing a New Family of Noncentrosymmetric Superconductor That Breaks TimeReversal Symmetry, Phys. Rev. Lett. 115, 267001 (2015).

[9] D. Singh, M. S. Scheurer, A. D. Hillier, and R. P. Singh, Time-reversal-symmetry breaking and unconventional pairing in the noncentrosymmetric superconductor $\mathrm{La}_{7} \mathrm{Rh}_{3}$ probed by $\mu$ SR, arXiv:1802.01533.

[10] R. P. Singh, A. D. Hillier, B. Mazidian, J. Quintanilla, J. F. Annett, D. M. Paul, G. Balakrishnan, and M. R. Lees, Detection of Time-Reversal Symmetry Breaking in the Noncentrosymmetric Superconductor $\mathrm{Re}_{6} \mathrm{Zr}$ Using MuonSpin Spectroscopy, Phys. Rev. Lett. 112, 107002 (2014).

[11] T. Shang, G. M. Pang, C. Baines, W. B. Jiang, W. Xie, A. Wang, M. Medarde, E. Pomjakushina, M. Shi, J. Mesot, H. Q. Yuan, and T. Shiroka, Nodeless superconductivity and time-reversal symmetry breaking in the noncentrosymmetric superconductor $\mathrm{Re}_{24} \mathrm{Ti}_{5}$, Phys. Rev. B 97, 020502(R) (2018).

[12] T. Shang, M. Smidman, S. K. Ghosh, C. Baines, L. J. Chang, D. J. Gawryluk, J. A. T. Barker, R. P. Singh, D. M. Paul, G. Balakrishnan, E. Pomjakushina, M. Shi, M. Medarde, A. D. Hillier, H. Q. Yuan, J. Quintanilla, J. Mesot, and T. Shiroka, Time-Reversal Symmetry Breaking in Re-Based Superconductors, Phys. Rev. Lett. 121, 257002 (2018).

[13] D. Singh, J. A. T. Barker, A. Thamizhavel, D. M. Paul, A. D. Hillier, and R. P. Singh, Time-reversal symmetry breaking in the noncentrosymmetric superconductor $\operatorname{Re}_{6} \mathrm{Hf}$ : Further evidence for unconventional behavior in the $\alpha$-Mn family of materials, Phys. Rev. B 96, 180501(R) (2017).

[14] A. P. Mackenzie and Y. Maeno, The superconductivity of $\mathrm{Sr}_{2} \mathrm{RuO}_{4}$ and the physics of spin-triplet pairing, Rev. Mod. Phys. 75, 657 (2003), and reference therein.

[15] R. Joynt and L. Taillefer, The superconducting phases of $\mathrm{UPt}_{3}$, Rev. Mod. Phys. 74, 235 (2002), and reference therein.

[16] Non-Centrosymmetric Superconductors, edited by E. Bauer and M. Sigrist (Springer Verlag, Berlin, 2012), Vol. 847.

[17] Y. Sungkit, Noncentrosymmetric superconductors, Annu. Rev. Condens. Matter Phys. 5, 15 (2014).

[18] M. Smidman, M. B. Salamon, H. Q. Yuan, and D. F. Agterberg, Superconductivity and spin-orbit coupling in non-centrosymmetric materials: A review, Rep. Prog. Phys. 80, 036501 (2017).

[19] H. Q. Yuan, D. F. Agterberg, N. Hayashi, P. Badica, D. Vandervelde, K. Togano, M. Sigrist, and M. B. Salamon, $S$-Wave Spin-Triplet Order in Superconductors Without Inversion Symmetry: $\mathrm{Li}_{2} \mathrm{Pd}_{3} \mathrm{~B}$ and $\mathrm{Li}_{2} \mathrm{Pt}_{3} \mathrm{~B}$, Phys. Rev. Lett. 97, 017006 (2006).

[20] M. Nishiyama, Y. Inada, and G.-q. Zheng, Spin Triplet Superconducting State Due to Broken Inversion Symmetry in $\mathrm{Li}_{2} \mathrm{Pt}_{3} \mathrm{~B}$, Phys. Rev. Lett. 98, 047002 (2007).

[21] I. Bonalde, W. Brämer-Escamilla, and E. Bauer, Evidence for Line Nodes in the Superconducting Energy Gap of Noncentrosymmetric $\mathrm{CePt}_{3} \mathrm{Si}$ from Magnetic Penetration Depth Measurements, Phys. Rev. Lett. 94, 207002 (2005).

[22] G. M. Pang, M. Smidman, W. B. Jiang, J. K. Bao, Z. F. Weng, Y. F. Wang, L. Jiao, J. L. Zhang, G. H. Cao, and H. Q.
Yuan, Evidence for nodal superconductivity in quasi-onedimensional $\mathrm{K}_{2} \mathrm{Cr}_{3} \mathrm{As}_{3}$, Phys. Rev. B 91, 220502(R) (2015).

[23] D. T. Adroja, A. Bhattacharyya, M. Telling, Y. Feng, M. Smidman, B. Pan, J. Zhao, A. D. Hillier, F. L. Pratt, and A. M. Strydom, Superconducting ground state of quasi-onedimensional $\mathrm{K}_{2} \mathrm{Cr}_{3} \mathrm{As}_{3}$ investigated using $\mu \mathrm{SR}$ measurements, Phys. Rev. B 92, 134505 (2015).

[24] E. Bauer, G. Hilscher, H. Michor, C. Paul, E. W. Scheidt, A. Gribanov, Y. Seropegin, H. Noël, M. Sigrist, and P. Rogl, Heavy Fermion Superconductivity and Magnetic Order in Noncentrosymmetric $\mathrm{CePt}_{3} \mathrm{Si}$, Phys. Rev. Lett. 92, 027003 (2004).

[25] E. M. Carnicom, W. Xie, T. Klimczuk, J. J. Lin, K. Górnicka, Z. Sobczak, N. P. Ong, and R. J. Cava, $\mathrm{TaRh}_{2} \mathrm{~B}_{2}$ and $\mathrm{NbRh}_{2} \mathrm{~B}_{2}$ : Superconductors with a chiral noncentrosymmetric crystal structure, Sci. Adv. 4, eaar7969 (2018).

[26] N. Kimura, K. Ito, H. Aoki, S. Uji, and T. Terashima, Extremely High Upper Critical Magnetic Field of the Noncentrosymmetric Heavy Fermion Superconductor $\mathrm{CeRhSi}_{3}$, Phys. Rev. Lett. 98, 197001 (2007).

[27] J. Chen, M. B. Salamon, S. Akutagawa, J. Akimitsu, J. Singleton, J. L. Zhang, L. Jiao, and H. Q. Yuan, Evidence of nodal gap structure in the noncentrosymmetric superconductor $\mathrm{Y}_{2} \mathrm{C}_{3}$, Phys. Rev. B 83, 144529 (2011).

[28] H. Kim, K. Wang, Y. Nakajima, R. Hu, S. Ziemak, P. Syers, L. Wang, H. Hodovanets, J. D. Denlinger, P. M. R. Brydon, D. F. Agterberg, M. A. Tanatar, R. Prozorov, and J. Paglione, Beyond triplet: Unconventional superconductivity in a spin$3 / 2$ topological semimetal, Sci. Adv. 4, eaao4513 (2018).

[29] Z. X. Sun, M. Enayat, A. Maldonado, C. Lithgow, E. Yelland, D. C. Peets, A. Yaresko, A. P. Schnyder, and P. Wahl, Dirac surface states and nature of superconductivity in noncentrosymmetric BiPd, Nat. Commun. 6, 6633 (2015).

[30] M. N. Ali, Q. D. Gibson, T. Klimczuk, and R. J. Cava, Noncentrosymmetric superconductor with a bulk threedimensional Dirac cone gapped by strong spin-orbit coupling, Phys. Rev. B 89, 020505(R) (2014).

[31] M. Sato and S. Fujimoto, Topological phases of noncentrosymmetric superconductors: Edge states, Majorana fermions, and non-Abelian statistics, Phys. Rev. B 79, 094504 (2009).

[32] Y. Tanaka, Y. Mizuno, T. Yokoyama, K. Yada, and M. Sato, Anomalous Andreev Bound State in Noncentrosymmetric Superconductors, Phys. Rev. Lett. 105, 097002 (2010).

[33] E. Bauer, G. Rogl, X.-Q. Chen, R. T. Khan, H. Michor, G. Hilscher, E. Royanian, K. Kumagai, D. Z. Li, Y. Y. Li, R. Podloucky, and P. Rogl, Unconventional superconducting phase in the weakly correlated noncentrosymmetric $\mathrm{Mo}_{3} \mathrm{Al}_{2} \mathrm{C}$ compound, Phys. Rev. B 82, 064511 (2010).

[34] V. K. Anand, A. D. Hillier, D. T. Adroja, A. M. Strydom, H. Michor, K. A. McEwen, and B. D. Rainford, Specific heat and $\mu \mathrm{SR}$ study on the noncentrosymmetric superconductor $\mathrm{LaRhSi}_{3}$, Phys. Rev. B 83, 064522 (2011).

[35] V. K. Anand, D. Britz, A. Bhattacharyya, D. T. Adroja, A. D. Hillier, A. M. Strydom, W. Kockelmann, B. D. Rainford, and K. A. McEwen, Physical properties of noncentrosymmetric superconductor $\mathrm{LaIrSi}_{3}$ : A $\mu \mathrm{SR}$ study, Phys. Rev. B 90, 014513 (2014).

[36] M. Smidman, A. D. Hillier, D. T. Adroja, M. R. Lees, V. K. Anand, R. P. Singh, R. I. Smith, D. M. Paul, and G. 
Balakrishnan, Investigations of the superconducting states of noncentrosymmetric $\mathrm{LaPdSi}_{3}$ and $\mathrm{LaPtSi}_{3}$, Phys. Rev. B 89, 094509 (2014).

[37] A. A. Aczel, T. J. Williams, T. Goko, J. P. Carlo, W. Yu, Y. J. Uemura, T. Klimczuk, J. D. Thompson, R. J. Cava, and G. M. Luke, Muon spin rotation/relaxation measurements of the noncentrosymmetric superconductor $\mathrm{Mg}_{10} \mathrm{Ir}_{19} \mathrm{~B}_{16}$, Phys. Rev. B 82, 024520 (2010).

[38] T. Shang, J. Philippe, J. A. T. Verezhak, Z. Guguchia, J. Z. Zhao, L.-J. Chang, M. K. Lee, D. J. Gawryluk, E. Pomjakushina, M. Shi, M. Medarde, H.-R. Ott, and T. Shiroka, Nodeless superconductivity and preserved timereversal symmetry in the noncentrosymmetric $\mathrm{Mo}_{3} \mathrm{P}$ superconductor, Phys. Rev. B 99, 184513 (2019).

[39] W. H. Lee, H. K. Zeng, Y. D. Yao, and Y. Y. Chen, Superconductivity in the $\mathrm{Ni}$ based ternary carbide $\mathrm{LaNiC}_{2}$, Physica (Amsterdam) 266C, 138 (1996).

[40] J. Chen, L. Jiao, J. L. Zhang, Y. Chen, L. Yang, M. Nicklas, F. Steglich, and H. Q. Yuan, Evidence for two-gap superconductivity in the non-centrosymmetric compound $\mathrm{LaNiC}_{2}$, New J. Phys. 15, 053005 (2013).

[41] Y. Hirose, T. Kishino, J. Sakaguchi, Y. Miura, F. Honda, T. Takeuchi, E. Yamamoto, Y. Haga, H. Harima, R. Settai, and Y. Ōnuki, Fermi surface and superconducting properties of non-centrosymmetric $\mathrm{LaNiC}_{2}$, J. Phys. Soc. Jpn. 81, 113703 (2012).

[42] I. Bonalde, R. L. Ribeiro, K. J. Syu, H. H. Sung, and W. H. Lee, Nodal gap structure in the noncentrosymmetric superconductor $\mathrm{LaNiC}_{2}$ from magnetic-penetration-depth measurements, New J. Phys. 13, 123022 (2011).

[43] J. F. Landaeta, D. Subero, P. Machado, F. Honda, and I. Bonalde, Unconventional superconductivity and an ambient-pressure magnetic quantum critical point in single-crystal $\mathrm{LaNiC}_{2}$, Phys. Rev. B 96, 174515 (2017).

[44] In an early report of the specific heat of $\mathrm{LaNiC}_{2}$, a $T^{2}$ dependence of $\mathrm{C} / \mathrm{T}$ was observed (indicating nodal SC) [39], but more recently exponential behavior of $C / T$ was reported, consistent with a fully gapped superconducting state [40,41]. Similar inconsistencies are also found from magnetic penetration depth $\lambda(T)$ measurements, where both a $T^{2}$ and an exponential temperature dependence have been reported $[40,42,43]$, consistent with the presence of point nodes and fully gapped behavior, respectively.

[45] J. Quintanilla, A. D. Hillier, J. F. Annett, and R. Cywinski, Relativistic analysis of the pairing symmetry of the noncentrosymmetric superconductor $\mathrm{LaNiC}_{2}$, Phys. Rev. B 82, 174511 (2010).

[46] Z. F. Weng, J. L. Zhang, M. Smidman, T. Shang, J. Quintanilla, J. F. Annett, M. Nicklas, G. M. Pang, L. Jiao, W. B. Jiang, Y. Chen, F. Steglich, and H. Q. Yuan, Two-Gap Superconductivity in $\mathrm{LaNiGa}_{2}$ with Nonunitary Triplet Pairing and Even Parity Gap Symmetry, Phys. Rev. Lett. 117, 027001 (2016).

[47] D. F. Agterberg, V. Barzykin, and L. P. Gor'kov, Conventional mechanisms for exotic superconductivity, Phys. Rev. B 60, 14868 (1999).
[48] S. Ghosh, J. F. Annett, and J. Quintanilla, Time-reversal symmetry breaking in superconductors through loop Josephson-current order, arXiv:1803.02618.

[49] W. Xie, P. R. Zhang, B. Shen, W. B. Jiang, G. M. Pang, T. Shang, C. Gao, M. Smidman, and H. Q. Yuan, CaPtAs: A new noncentrosymmetric superconductor, Sci. China Phys. Mech. Astron. 63, 237412 (2020).

[50] See the Supplemental Material at http://link.aps.org/ supplemental/10.1103/PhysRevLett.124.207001 for details on the measurements of the upper critical field, the analyses of superfluid density, specific heat, and ZF- $\mu$ SR data, and Refs. [51-52,54-55] therein.

[51] M. Tinkham, Introduction to Superconductivity, 2nd ed. (Dover Publications, Mineola, NY, 1996).

[52] N. R. Werthamer, E. Helfand, and P. C. Hohenberg, Temperature and purity dependence of the superconducting critical field, $H_{c 2}$. III. Electron spin and spin-orbit effects, Phys. Rev. 147, 295 (1966).

[53] E. H. Brandt, Properties of the ideal Ginzburg-Landau vortex lattice, Phys. Rev. B 68, 054506 (2003).

[54] H. Padamsee, J. E. Neighbor, and C. A. Shiffman, Quasiparticle phenomenology for thermodynamics of strongcoupling superconductor, J. Low Temp. Phys. 12, 387 (1973).

[55] F. Bouquet, Y. Wang, R. A. Fisher, D. G. Hinks, J. D. Jorgensen, A. Junod, and N. E. Phillips, Phenomenological two-gap model for the specific heat of $\mathrm{MgB}_{2}$, Europhys. Lett. 56, 856 (2001).

[56] R. Kubo and T. Toyabe, A stochastic model for low field resonance and relaxation, in Magnetic Resonance and Relaxation, edited by R. Blinc (North-Holland, Amsterdam, 1967), pp. 810-823.

[57] A. Yaouanc and P. D. de Réotier, Muon Spin Rotation, Relaxation, and Resonance: Applications to Condensed Matter (Oxford University Press, Oxford, 2011).

[58] A. Bhattacharyya, D. T. Adroja, K. Panda, S. Saha, T. Das, A. J. S. Machado, O. V. Cigarroa, T. W. Grant, Z. Fisk, A. D. Hillier, and P. Manfrinetti, Evidence of a Nodal Line in the Superconducting Gap Symmetry of Noncentrosymmetric $\mathrm{ThCoC}_{2}$, Phys. Rev. Lett. 122, 147001 (2019).

[59] K. V. Samokhin, E. S. Zijlstra, and S. K. Bose, $\mathrm{CePt}_{3} \mathrm{Si}$ : An unconventional superconductor without inversion center, Phys. Rev. B 69, 094514 (2004).

[60] K.-W. Lee and W. E. Pickett, Crystal symmetry, electronphonon coupling, and superconducting tendencies in $\mathrm{Li}_{2} \mathrm{Pd}_{3} \mathrm{~B}$ and $\mathrm{Li}_{2} \mathrm{Pt}_{3} \mathrm{~B}$, Phys. Rev. B 72, 174505 (2005).

[61] P. A. Frigeri, D. F. Agterberg, A. Koga, and M. Sigrist, Superconductivity Without Inversion Symmetry: MnSi Versus $\mathrm{CePt}_{3} \mathrm{Si}$, Phys. Rev. Lett. 92, 097001 (2004).

[62] A.P. Schnyder and P. M. R. Brydon, Topological surface states in nodal superconductors, J. Phys. Condens. Matter 27, 243201 (2015).

[63] M. Sato and Y. Ando, Topological superconductors: A review, Rep. Prog. Phys. 80, 076501 (2017). 